\title{
SUBSTATE AND SMSA PERSONAL INCOME INEQUALITY AND REGIONAL DEVELOPMENT
}

\author{
Orley M. Amos, Jr.*
}

\section{Introduction}

The relationship between income inequality and regional (national) development has been examined by Kuznets $(1955,1963,1973)$, Williamson $(1965,1980)$, Rice and Sale (1975), and Gunther and Leathers (1975). In particular, the inverted- $U$ pattern of income inequality has been subjected to extensive empirical testing by Williamson (1965). In a related line of research, the growth pole theory of regional development first discussed by Perroux (1955) and later by Hansen (1967) and Lasuen (1969), has been empirically examined by Lewis and Prescott (1972), Martin (1978, 1979, 1979b), and Martin and Graham (1980). Growth pole theory offers one explanation for observable patterns of regional development, including income inequality. This paper examines income inequality within a growth pole context testing whether growth poles are a necessary or merely sufficient condition for regional development.

Theoretically, growth poles can stimulate and sustain regional development, but it is possible for development to occur without dependence on growth poles. If growth poles are not a necessary condition for development, then regional development policies in the United States, and throughout the world, can consider other alternatives. While Martin (1978) has demonstrated the effectiveness of the growth pole oriented policies of the Economic Development Administration, development could be promoted, perhaps more effectively, by stimulating growth in nongrowth centers or rural areas.

To test the relationships between regional development and growth pole theory, patterns of income inequality between states by city size are examined. The next section identifies expected patterns of income inequality if growth pole theory is correct. The third and fourth sections present results of empirical

- Oklahoma State University. The author wishes to acknowledge the helpful comments of James Hite and two anonymous reviewers. tests of the expected patterns for substate and SMSA data respectively. The final section concludes this paper.

\section{Expected Patterns of Income Inequality}

Simon Kuznets first introduced the inverted-U pattern of income inequality in 1955 , with further discussion in later papers $(1963,1973)$. The inverted- $U$ hypothesis states that income inequality increases, then decreases, as development proceeds. In initial stages of development, wealthier members of society accumulate capital, and otherwise start the economy towards development, which subsequently leads to further increases in their relative income. However, as development spreads throughout the economy, capital accumulation raises productivity, and incomes of the poorest workers increase. At the same time, new jobs for the unemployed are provided.

An extensive test of the inverted-U hypothesis for regional income variation was performed by Williamson (1965) using crosssectional and time series data for countries in various stages of development, and crosssectional data for the United States. His analysis generally provided support for the inverted-U hypotheses. A more thorough examination of regional income variation in the United States by Amos (1985) indicated that the inverted-U may be an incomplete representation of the pattern of income inequality. The inverted-U hypotheses implicitly states that once income inequality declines, it remains relatively stable at a low level of inequality. However, a more complete pattern would include, at the very least, a second phase of increasing inequality. In this study an extension of the inverted-U hypotheses is postulated, stating that income inequality follows a pattern of increase-decrease-increase. It is hypothesized that after a phase of decreasing income inequality, the most developed states once again experience increasing income inequality.

Figure 1 illustrates the two competing 


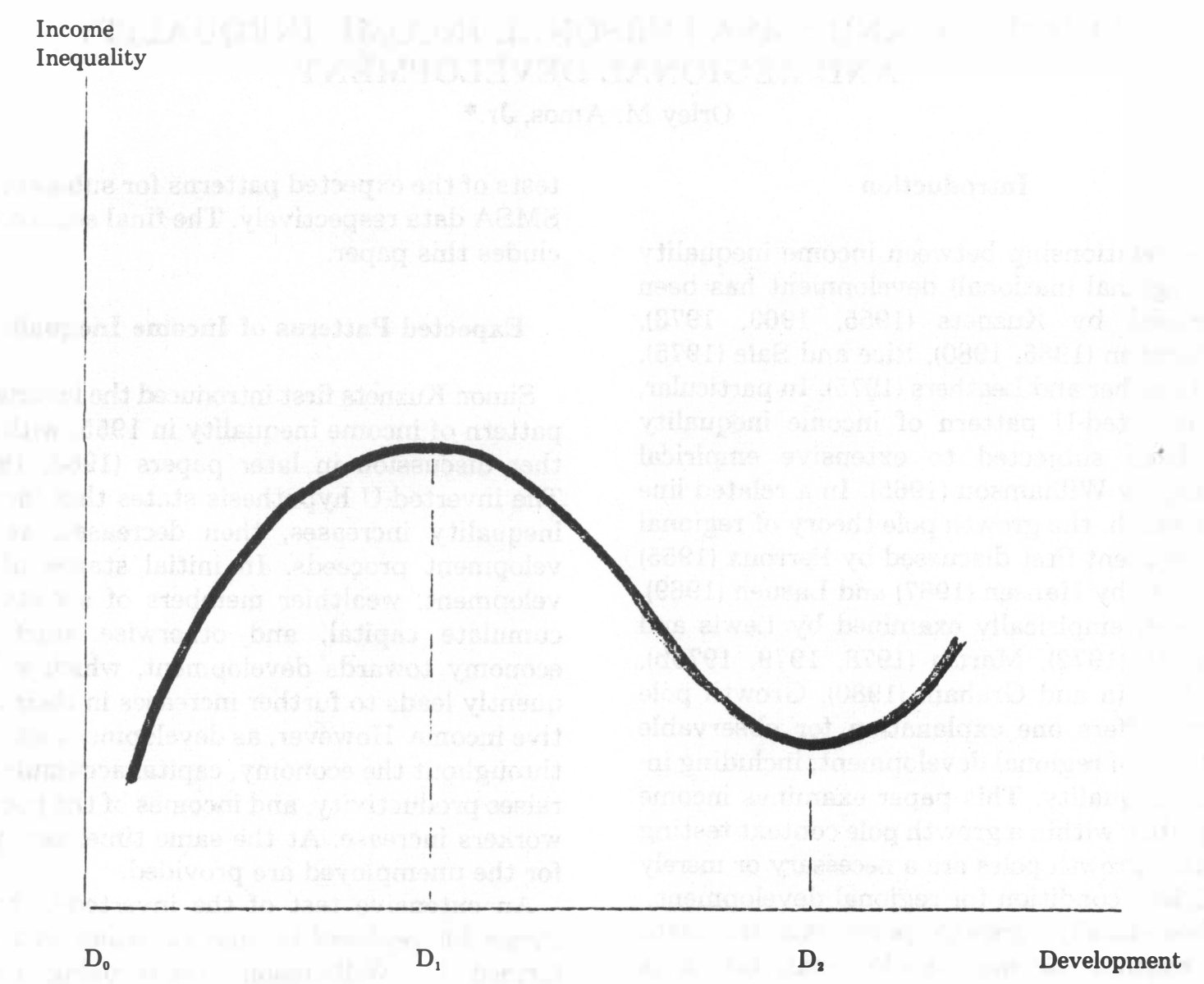

Figure 1

hypotheses to be tested in this study, the inverted- $U$ and an augmented inverted- $U$. The inverted- $U$ pattern exists between development levels $D_{0}$ and $D_{2}$, in which income inequality increases up to development level $D_{1}$, and between $D_{1}$ and $D_{2}$, in which inequality decreases. In this study it is hypothesized that beyond development level $D_{2}$, inequality once again increases.

At this point an important distinction should be noted. Kuznets' original paper in 1955 and studies by Rice and Sale (1975) and Gunther and Leathers (1975), are concerned with the distribution of personal income, or personal income inequality. However, Williamson used the spatial distribution of per capita income, or regional income variation. In a related study Amos (1983) notes that regional income variation and personal income inequality are highly correlated at some stages of development but not at others. However, reasoning behind increasing regional income variation identified by Amos (1985) also applies to personal income inequality. Regional income variation increases beyond $\mathrm{D}_{2}$ in Figure 1 as the per capita income in some lower income regions catch up to, and surpass, initially higher income regions. The same justification also applies to personal income inequality, as some lower income groups catch up to, and surpass, initially higher income individuals. This paper examines personal income inequality and the substate level to determine if it follows a pattern of increase-decrease-increase.

\section{Growth Pole Theory and the Inverted-U Hypothesis}

Perroux (1955) first discussed growth pole theory and regional development. He noted that development appears first at nodes or poles, and subsequently spreads to the periphery. Growth pole theory easily explains the inverted-U pattern (increase-decrease) of both personal income inequality and regional in- 
come variation. In the early stages of development capital accumulates at the growth pole, resulting in concentrations of capital ownership, both spatially and personally, raising incomes at the pole, and increasing both types of inequality. Development at the pole spreads to the periphery at increasing rates as transportation and communication systems between the growth pole and the surrounding area improve. More efficient transportation and communication systems reduce the attraction of the growth pole, and the relative rate of capital accumulation decreases at the growth pole while increasing in the periphery. This process reduces both regional and personal income inequality.

The augmented inverted- $U$ pattern (increasedecrease-increase) can also be explained by growth pole theory. The forces which induce capital accumulation in the periphery not only enable the surrounding area to catch up to the growth pole, but also to surpass it. While it is not likely that development in the entire surrounding area surpasses that of the growth, it is possible for this to happen to portions of the surrounding area. In essence secondary growth centers might be established. The most obvious case of this phenomenon since 1950 has been suburbanization of residential, commercial and manufacturing activities, which were formerly the exclusive domain of major urban areas (Mills, 1980, pp. 38-49).

As secondary growth centers emerge, both regional and personal income inequality increase for the exact reasons that the initial growth pole caused increased inequality. The only difference between the initial and secondary growth poles is the overall level of development existing at the time the process begins.

\section{Substate Patterns}

One last piece must be added to the puzzle before identifying testable hypotheses. Kuznets' inverted-U was designed and tested at the national or state level. The importance of this geographic level is the existence within each area of variation in population and capital concentration. That is, all areas previously tested have contained both urban and rural areas. Further tests of growth pole theory, the inverted-U and the augmented inverted-U can be performed by examining urban areas in different stages of development. If development follows the inverted-U pattern, but not as a consequence of growth poles, then urban areas, as well as states and nations, should follow the pattern. If regional development is due $e x$ clusively to the growth pole process, then there is no reason for urban areas to follow the inverted-U, since each is only a part, albeit an integral part, of a larger region.

In addition it was indicated that emergence of secondary growth centers can explain the redivergence in income inequality in more developed states. However, if more developed urban areas also experience the increased inequality, this indicates that emergence of secondary growth centers may not be an appropriate explanation. As indicated by Amos (1985), increased inequality in later stages may be a natural sequence in the overall course of development.

Hypothesized Relationships Between Income Inequality and Development

Three patterns of income inequality are possible, based upon the previous discussion.

1. The inverted-U. The inverted-U pattern is supported if income inequality decreases with higher levels of development. If this pattern is identified upon analysis of urban areas exclusively, with no upturn evident, it indicates secondary growth poles are necessary to explain increased income inequality at the state level.

2. The augmented inverted-U. Since the United States is to the right of $D_{1}$ in Figure 1, an indication of increased inequality at higher levels of development supports this hypothesis. If this pattern is identified for various substate regions, it indicates secondary growth poles are not necessary to explain increased income inequality. The divergence of income in more developed states may be due to various unidentified factors which are causing the pattern to occur in substate regions.

3. No relationship. If no pattern is identified between income inequality and development in urban areas, this supports growth pole theory. If the inverted-U pattern exists at the state level, but not at the urban level, this implies urban and rural areas are necessary within a region before the inverted-U pattern appears. 
Therefore both the growth pole and surrounding area are required for this pattern. A growth pole produces inequality between itself and the surrounding area, and not exclusively within itself.

These three hypotheses are tested using cross sectional income inequality data from the 1970 U.S. Census of Population. Although the thrust of this study is to identify patterns of income inequality over time, cross sectional data can be satisfactorily employed to pursue this objective. First, the census provides a wider range of data needed to test these hypotheses than is available in time series form. Second, states have a wide range of variation in their levels of development, with as much cross sectional variation in the development among states in 1970 as for single states in recent years of data availability.

\section{Results: Substate Data}

The first test of the three hypotheses presented above employs substate data. Table

Table 1

Regression Analysis of Gini Coefficients and Per Capita Income for Substate Areas, 1970

\begin{tabular}{|c|c|c|c|c|c|c|}
\hline $\begin{array}{l}\text { Substate } \\
\text { Area }\end{array}$ & $\begin{array}{c}\text { Equation } \\
\text { Number }\end{array}$ & Constant & $\begin{array}{l}\text { Per Capita } \\
\text { Income }\end{array}$ & $\begin{array}{c}\text { Per Capita } \\
\text { Income-Squared }\end{array}$ & $\mathbf{R}^{2}$ & F.Value \\
\hline \multirow[t]{2}{*}{ Total Urban } & (1) & 1.061 & $\begin{array}{l}-0.120 \\
(4.189)^{* * * *}\end{array}$ & $\begin{array}{c}0.00500 \\
(3.937)^{* * * *}\end{array}$ & 0.390 & $15.052^{* * *}$ \\
\hline & ( 2) & 0.433 & $\begin{array}{l}-0.008 \\
(3.349)^{* *}\end{array}$ & & 0.189 & $11.219 * * *$ \\
\hline \multirow[t]{2}{*}{ Urbanized Areas } & (3) & 1.011 & $\begin{array}{l}-0.109 \\
(2.202)^{*}\end{array}$ & $\begin{array}{c}0.00411 \\
(2.053)^{*}\end{array}$ & 0.221 & $6.248^{* *}$ \\
\hline & (4) & 0.431 & $\begin{array}{l}-0.007 \\
(2.780)^{* *}\end{array}$ & & 0.147 & $7.729 * * *$ \\
\hline \multirow[t]{2}{*}{ Central Cities } & (5) & 1.387 & $\begin{array}{l}-0.172 \\
(3.382)^{* *}\end{array}$ & $\begin{array}{c}0.00709 \\
(3.182)^{* *}\end{array}$ & 0.293 & $9.099 * * *$ \\
\hline & (6) & 0.474 & $\begin{array}{l}-0.000 \\
(2.590)^{*}\end{array}$ & & 0.130 & $6.710^{* *}$ \\
\hline \multirow[t]{2}{*}{ Urban Fringe } & ( 7) & 0.477 & $\begin{array}{c}-0.026 \\
(1.208)\end{array}$ & $\begin{array}{l}0.00104 \\
(1.158)\end{array}$ & 0.038 & 0.856 \\
\hline & (8) & 0.334 & $\begin{array}{c}-0.001 \\
(0.607)\end{array}$ & & 0.008 & 0.369 \\
\hline \multirow[t]{2}{*}{ Medium Places } & (9) & 1.075 & $\begin{array}{l}-0.124 \\
(6.505)^{* * *}\end{array}$ & $\begin{array}{l}0.00509 \\
(6.192)^{* * * *}\end{array}$ & 0.528 & $26.330 * * *$ \\
\hline & (10) & 0.434 & $\begin{array}{l}-0.008 \\
(2.838)^{* *}\end{array}$ & & 0.144 & $8.053^{* *}$ \\
\hline \multirow[t]{2}{*}{ Small Places } & (11) & 1.152 & $\begin{array}{l}-0.151 \\
(5.088)^{* * * *}\end{array}$ & $\begin{array}{l}0.00692 \\
(4.573)^{* * * *}\end{array}$ & 0.637 & $41.149 * * *$ \\
\hline & (12) & 0.498 & $\begin{array}{l}-0.000 \\
(6.587)^{* * * *}\end{array}$ & & 0.475 & $43.388^{* * *}$ \\
\hline \multirow[t]{2}{*}{ Total Rural } & (13) & 0.654 & $\begin{array}{l}-0.050 \\
(4.753)^{* * *}\end{array}$ & $\begin{array}{l}0.00199 \\
(3.862)^{* * * *}\end{array}$ & 0.559 & $29.762 * * *$ \\
\hline & (14) & 0.457 & $\begin{array}{l}-0.010 \\
(5.881)^{* * *}\end{array}$ & & 0.419 & $34.581 * * *$ \\
\hline \multirow[t]{2}{*}{ Rural Nonfarm } & (15) & 0.666 & $\begin{array}{l}-0.056 \\
(5.434)^{* * *}\end{array}$ & $\begin{array}{l}0.00227 \\
(4.506)^{* * *}\end{array}$ & 0.596 & $31.695^{* * *}$ \\
\hline & (16) & 0.446 & $\begin{array}{l}-0.010 \\
(5.472)^{* * *}\end{array}$ & & 0.405 & $29.942 * * *$ \\
\hline \multirow[t]{2}{*}{ Rural Farm } & (17) & 0.572 & $\begin{array}{r}-0.029 \\
(1.714)\end{array}$ & $\begin{array}{l}0.00132 \\
(1.507)\end{array}$ & 0.098 & 2.347 \\
\hline & (18) & 0.457 & $\begin{array}{r}-0.004 \\
(1.554)\end{array}$ & & 0.051 & 2.355 \\
\hline
\end{tabular}

* Statistically significant at $\alpha=0.05$.

** Statistically significant at $\alpha=0.01$.

*** Statistically significant at $\alpha=0.001$. 
1 presents regression results for nine substate regions: total urban (TU), urbanized areas (UA), central cities (CC), urban fringes (UF), medium size regions with population over 10,000 but not urbanized (MC), small size regions with population between 2,500 and 10,000 (SC), total rural (TR), rural nonfarm (RNF), and rural farm (RF). For each substate region both linear and quadratic regressions between the Gini coefficient measure of personal income inequality and per capita income are presented. ${ }^{1}$.

Several important points are evident in Table 1. First, in every equation per capita income is inversely related to the Gini coefficient. In every equation except that for the UF and RF substate areas, the per capita income coefficient is significant at $\alpha<0.05$. Clearly this result is supportive of the inverted-U hypothesis. As per capita income increases, income inequality is reduced, regardless of the substate area. This indicates the development/inequality phenomenon transcends the level of regionalization. It is evident that, at the very least, the inverted-U pattern holds not just for nations or states, as has been tested up to this point, but also for smaller regionalizations of states. However, is the inverted- $U$ or the augmented inverted- $U$ pattern indicated by the substate results?

The quadratic equation for each substate region supports the augmented inverted- $U$ hypothesis. The coefficient of the square of per capita income is positive and significant at $\alpha \leq$ 0.05 , again for every region except UF and RF. The negative sign for the per capita income coefficient and the positive sign for the per capita income-squared coefficient, indicate that substate regions are in the U-shaped portion of Figure 1 surrounding $D_{2}$, which supports the augmented inverted- $U$ hypothesis.

The pattern, clearly depicted by every quadratic equation (except for UF and RF in Table 1) is very indicative of the development/inequality process. The substate regions have increasing inequality in the most developed areas, indicating that the development/ inequality pattern goes beyond national or state level. By all indications the interaction between a highly urbanized, densely concentrated growth pole and a more sparse periphery, is not a necessary condition for increasing inequality to occur. According to growth pole theory, the emergence of sec- ondary growth poles, i.e. suburbanization, can explain increasing regional income inequality at the state level. However, in that the substate regions follow the augmented inverted- $U$ pattern, the growth pole explanation is not supported. Of the three hypotheses presented above, (1) the inverted- $U,(2)$ the augmented inverted- $U$, and (3) no relationship, clearly the second hypothesis is supported by Table 1 .

The quadratic equations in Table 1 present additional information worthy of analysis. The points at which each equation reaches its minimum Gini coefficient values are presented in Table 2. In the previously cited study of spatial and personal income inequality, Amos (1983) speculated that personal income inequality is reduced as development increases, but only to a certain level. The level Amos empirically identifies as a minimum Gini coefficient is about 0.300 . There is a degree of consistency between the minimum value of the Gini coefficient for each equation and the results identified by Amos. However, while the average of the minimum values in Table 2 is 0.331 , the range is from 0.288 for UA to 0.413 for RF. In that the range of raw Gini coefficients data for the substate regions is from 0.256 to 0.428 , the values in Table 2 appear to have too much variation to indicate any global minimum.

However, a distinct pattern is depicted by the minimum values in Table 2 . The substate areas used in this analysis can be divided into different exhaustive sets. That is each state is divided into total urban (TU) and total rural (TR) areas. The TU region can be further divided into UA, MC, and SC. The UA substate region can be divided into $\mathrm{CC}$ and UF. The TR area

Table 2

Minimum Gini Coefficient Values of Quadratic Equations in Table 1

\begin{tabular}{cc}
\hline Substate Area & Minimum Gini Coefficient \\
\hline Total Urban & 0.341 \\
Urbanized Areas & 0.288 \\
Central Cities & 0.344 \\
Urban Fringe & 0.315 \\
Medium Places & 0.320 \\
Small Places & 0.328 \\
Total Rural & 0.340 \\
Rural Non-Farm & 0.321 \\
Rural Farm & 0.413 \\
\hline
\end{tabular}


can be divided into RNF and RF.

Depending on the level of disaggregation of substate groups, several points are evident. Looking first at the most aggregate regions, TU and TR have nearly identical minimum Gini coefficient values, 0.341 and 0.340 , respectively. This could be an estimate of a global minimum, if one exists. However, analysis of the more disaggregate substate regions indicates a different interpretation.

The second level of disaggregation, containing substate groups UA, MC, SC, RNF, and $\mathrm{RF}$, indicates a distinct pattern. From RF to UA the minimum Gini coefficient values in Table 2 continually decrease, except for a slight increase from RNF to SC. The third level of disaggregation, similar to the second, except UA is divided into CC and UF, shows a slightly different pattern. Instead of a constant decrease in minimum Gini coefficient values from the least to most urbanized substate regions, there is an increase from UF to CC. In effect the pattern depicted by the minimum Gini coefficients is U-shaped, decreasing from RNF to UF, then increasing to CC.

The degree of urbanization, and consequently the density of population and economic activity, indicates why this pattern might occur. For a given level of development, an area that has a greater concentration of economic activity will have lower transportation and communication costs. Reduction of transportation and communication costs is often cited as a fundamental reason for existence of urban areas. As these costs are relatively lower, the allocation of goods and services in the region is more efficient. Consequently income inequality at a given level of development could be relatively less, as allocation efficiency is enhanced. In essence the minimum Gini coefficient values in Table 2, if they all correspond to the same level of development, are lower for more urbanized areas because the allocation process is more efficient.

If this interpretation is correct, the increase from UF to CC would be expected. Given that congestion is greater in central cities, transportation costs would be greater, inhibiting the allocation process and preventing income inequality from being as low as it is in the UF.

If the minimum Gini coefficient values for substate augmented inverted-U curves, together form a U-shaped, upturned pattern, the equivalence of TU and TR minimums has a dif- ferent interpretation than earlier presented. Instead of the TU and TR minimums implying a universal minimum, they might be, in fact, two points on the upslope and downslope of the U-shaped pattern depicted by the other substate minimum values.

\section{Results: SMSA Data}

Four differences exist between substate and SMSA data that are both advantageous and disadvantageous. First, SMSA data provide more observations than substate data (243 versus 50$).{ }^{2}$ Second, more information is available at the SMSA level than at the substate level. Of particular importance is a cost of living index that is used to obtain a measure of real per capita income. ${ }^{3}$ Third, each SMSA constitutes a single continuous region. The substate regions are based on the total population of a state that lives in a certain city-size. As a more geographically dispersed population is included in a substate group, greater heterogeneity is possible, in particular with respect to factors other than per capita income that might affect development. Fourth, in that SMSAs are contiguous county delineations, a single SMSA often includes the entire spectrum of urbanization, from central city to rural farm. Therefore the SMSA data base is closer in structure to heterogeneous nations and states than the substate data base. As in national and state income inequality studies that have been undertaken, there is a greater chance of picking up the growth pole/periphery relationship.

Table 3 presents regression analysis using SMSA data. Like the substate data, both simple linear and quadratic regression equations are presented, first with nominal per capita income and subsequently with real per capita income. The SMSA results are consistent with the substate results. In every equation income is inversely related to the Gini coefficient and the square of income is positively related, indicating a U-shaped pattern consistent with the portion of Figure 1 surrounding $D_{2}$. For both nominal and real per capita income, the quadratic equation has a significantly better fit than the simple linear equation.

In both quadratic equations (19) and (21), the minimum Gini coefficient is consistent with results in Table 2. For nominal per capita income the minimum Gini coefficient is 0.322 , 
Table 3

Regression Analysis of Gini Coefficients for SMSAs, 1970

\begin{tabular}{|c|c|c|c|c|c|c|c|c|}
\hline \multicolumn{2}{|c|}{ Constant } & $\begin{array}{l}\text { Per Capita } \\
\text { Income }\end{array}$ & $\begin{array}{c}\text { Per Capita } \\
\text { Income-Squared }\end{array}$ & Population & $\begin{array}{l}\text { Population- } \\
\text { Squared }\end{array}$ & $\begin{array}{l}\text { Real Per Capita } \\
\text { Income }\end{array}$ & \multicolumn{2}{|c|}{ Income-Squared (F-Value) } \\
\hline (19) & 0.611 & $\begin{array}{l}-0.143 \\
(8.772)^{* * *}\end{array}$ & $\begin{array}{c}0.0177 \\
(7.520)^{* * *}\end{array}$ & & & & & $\begin{array}{l}0.298 \\
(51.035)^{* * * *}\end{array}$ \\
\hline (20) & 0.415 & $\begin{array}{l}-0.023 \\
(6.082)^{* * *}\end{array}$ & & & & & & $\begin{array}{c}0.133 \\
(36.993)^{* * *}\end{array}$ \\
\hline (21) & 0.746 & & & & & $\begin{array}{l}-0.241 \\
(6.657)^{* * *}\end{array}$ & $\begin{array}{l}0.0350 \\
(5.952)^{* * *}\end{array}$ & $\begin{array}{c}0.224 \\
(34.730)^{* * * *}\end{array}$ \\
\hline$(22)$ & 0.425 & & & & & $\begin{array}{l}-0.027 \\
(5.457)^{* * *}\end{array}$ & & $\begin{array}{c}0.110 \\
(29.783)^{* * *}\end{array}$ \\
\hline (23) & 0.649 & $\begin{array}{l}-0.1576 \\
(9.718)^{* * *}\end{array}$ & $\begin{array}{c}0.0189 \\
*(8.212)^{* * *}\end{array}$ & $\begin{array}{l}0.0110 \\
(2.969)^{* *}\end{array}$ & $\begin{array}{c}-0.00052 \\
(1.298)\end{array}$ & & & $\begin{array}{c}0.351 \\
(32.135)^{* * * *}\end{array}$ \\
\hline (24) & 0.753 & & & $\begin{array}{l}0.0031 \\
(8.240)^{* * * *}\end{array}$ & $\begin{array}{l}0.00009 \\
(0.200)\end{array}$ & $\begin{array}{l}-0.244 \\
(6.770)^{* * *}\end{array}$ & $\begin{array}{l}0.0351 \\
(6.005)^{* * *}\end{array}$ & $\begin{array}{c}0.242 \\
(18.955)^{* * * *}\end{array}$ \\
\hline
\end{tabular}

**Statistically significant at $\alpha=0.01$.

*** Statistically significant at $\alpha=0.001$.

and for real per capita income the minimum is 0.331 . In that SMSAs are contiguous counties, unlike the substate observations, there is no substate region in Tables 1 or 2 directly comparable to SMSAs. However, the TU region is perhaps, conceptually, the closest. The TU region has a minimum Gini coefficient value of 0.341 .

It was speculated earlier that the minimum Gini coefficient values for different substate regions form a U-shaped pattern due to relative transportation costs in areas of different density. This hypothesized relationship is also tested with SMSA data. Equations (23) and (24) in Table 3 include SMSA population and SMSA population-squared as independent variables in both the nominal and real quadratic equations, respectively.

If the hypothesized relationship is correct, then a U-shape should be indicated by the population and population-squared variables, i.e., negative and positive coefficients, respectively. This is not indicated by equations (23) and (24). The population variable is significant but positive, and the population-squared variable is not statistically significant. Clearly a U-shaped pattern is not indicated. However, it is possible the SMSA data captures only the upslope of the U-pattern, since the data is urban oriented, excluding medium places, small places and rural areas that are not in SMSA counties.

\section{Conclusion}

Three primary conclusions are obtained from the results of this study. First, there is explicit support for the augmented inverted- $U$ hypothesis. In analysis of substate areas and SMSAs, an increase in income inequality of the most developed areas is indicated by the regression analysis. These results imply an augmented inverted-U pattern for substate regions, homogeneous in city-size, as well as heterogeneous state and national areas containing urban and rural regions. Clearly the prevalence of this pattern necessitates additional investigation into the underlying causes. Are the same factors causing increased inequality for more developed urban areas also causing this pattern for more developed rural areas? A recent study by Maxwell and Wilson (1983) concerning structural change provides an important line of investigation relating to potential causes.

The second major conclusion reinforces the need for additional investigation. In that augmented inverted-U patterns are identified for all substate areas from central city to rural non-farm (except urban fringe and rural farm), it implies that the interaction between urban (growth pole) and rural (periphery) areas is not needed to explain increasing income inequality. If the growth pole process is not causing the increase, further investigation into the cause(s) is needed.

A third conclusion of this study is the observed relationship between the minimum Gini coefficient values and the degree of urbanization of the substate regions. While this is an 
empirical relationship, it does have theoretical support when transportation costs are considered. To extend this line of thought and add further speculation, transportation costs may be a significant factor in the general augmented inverted- $U$ upturn identified on the state and substate level.

\section{FOOTNOTES}

${ }^{1}$ Gini coefficient and per capita income data are obtained from the 1970 Census of Population.

${ }^{2}$ SMSA per capita means, population, and Gini coefficient data are obtained from the 1970 Census of Population.

The cost of living index for SMSAs was obtained from Ben-Chieh Liu's study of Quality of Life Indicators (1975).

\section{REFERENCES}

Amos, O. "The Relationship Between Regional Income Inequality, Personal Income Inequality, and Development." Regional Science Perspectives, 13 (1983), 3-14.

Amos, O. "Regional Income Variation in The Latter Stages of Development: Evidence from the United States." Presented at the Southern Economics Association meetings, Dallas, Texas, November 24-26, 1985.

Gunter, W. D. and C. G. Leathers. "Trends in Income Inequality in the South, 1950 to 1970." Growth and Change 6 (1975), 19-22.

Hansen, N. M. "Development Pole Theory in a Regional Context.” Kyklos, 20 (1967), 709-725.

Kuznets, S. "Economic Growth and Income Inequality." American Economic Review, 45 (1955), 1-28.

Kuznets, S. "Quantitative Aspects of the Economic Growth of Nations VIII: Distribution of Income by Size." Economic Development and Cultural Change, 11 (1963), 1-71.

Kuznets, S. "Modern Economic Growth: Findings and Reflections." American Economic Review, 63 (1973), 247-258.
Lasuien, J. R. “On Growth Poles.” Urban Studies (1969), 137-161.

Lewis, W. C. and J. Prescott. "Urban-Regional Development and Growth Centers: An Econometric Approach." Journal of Regional Science, 12 (1972), 57-70.

Liu, B. C. Quality of Life Indicators in U.S. Metropolitan Areas, 1970, U.S. Environmental Protection Agency, Washington, D.C., 1975.

Martin, R. C. "An Empirical Inquiry into the Effectiveness of the Growth Center Approach to Regional Economic Development." The Review of Regional Studies, 8 (1978), 1-19.

Martin, R. C. "Legislation Versus Administration: An Empirical Note on Federal Regional Development Programs." Growth and Change, 10 (1979a), 46-49.

Martin, R. C. "Federal Regional Development Programs and U.S. Problem Areas." Journal of Regional Science, 19 (1979b), 157-170.

Martin, R. C. and R. E. Graham Jr. "The Impact of Economic Development Administration Programs: Some Empirical Evidence." Review of Economics and Statistics, 62 (1980), 52-62.

Maxwell, P. and M. Wilson. "Structural Changes in Regional Labor Markets: the Australian Experience of the Seventies." Review of Regional Studies, 13 (1983) 2, 32-41.

Mills, E. S. Urban Economics, Second Edition. Scott, Foresmand and Co. (Glenview, Illinois: 1980).

Perroux, F. "Note sur la notion de "pole de croissance?" Economie Appliqe", (1955), 307-320.

Rice, G. R. and T. S. Sale. "Size Distribution of Income in Louisiana and Other Southern States." Growth and Change, 6 (1978), 20-33.

Williamson, Jeffrey G. "Regional Inequality and the Process of National Development: A Description of the Patterns." Economic Development and Cultural Change, 13 (1965), 1-45.

Williamson, Jeffrey G. "Unbalanced Growth, Inequality, and Regional Development: Some Lessons from U.S. History." In Arnold, Victor L. (ed.), Alternatives to Confrontation, Lexington Books (Lexington Books: 1980), 3-62. 